\title{
Yizhi decoction as a therapy for vascular dementia: A meta- analysis
}

\author{
Zhengjun Xin ${ }^{1}$, Sheng $\mathrm{Li}^{2 *}$ \\ ${ }^{1}$ College of Pharmacy, ${ }^{2}$ College of Management, Chengdu University of Traditional Chinese Medicine, Chengdu 611137, China \\ *For correspondence: Email: soonl@sina.com; Tel: 028-61800237
}

Sent for review: 16 May 2018

Revised accepted: 23 November 2018

\begin{abstract}
Purpose: Vascular dementia (VD) constitutes a heavy burden in health care systems in ageing societies. This review was aimed at assessing the effectiveness of Yizhi decoction against VD.

Methods: Five computerized databases were searched. A total of 262 publications were retrieved. Jadad evaluation was used to analyze the quality of the literature. Random methods, random allocation concealment and blinding, withdrawal, exiting and other aspects were included. RevMan 5.3 was used for meta-analysis.

Results: There were 1045 patients, consisting of 527 cases in the treatment group (Yizhi decoction group) and 518 cases in the control group. Risk ratio revealed that Yizhi decoction was significantly effective against VD, while the weighted by mean difference demonstrated that Yizhi decoction improved the MMSE score of patients.

Conclusion: Yizhi decoction improves the quality of daily life and mental state in VD patients. However, more rigorous and scientific case studies are still needed for further confirmation to strengthen and support these findings.
\end{abstract}

Keywords: Vascular dementia, Yizhi decoction, Meta-analysis

This is an Open Access article that uses a funding model which does not charge readers or their institutions
for access and distributed under the terms of the Creative Commons Attribution License
(http://creativecommons.org/licenses/by/4.0) and the Budapest Open Access Initiative
(http://www.budapestopenaccessinitiative.org/read), which permit unrestricted use, distribution, and
reproduction in any medium, provided the original work is properly credited.
Tropical Journal of Pharmaceutical Research is indexed by Science Citation Index (SciSearch), Scopus,
International Pharmaceutical Abstract, Chemical Abstracts, Embase, Index Copernicus, EBSCO, African
Index Medicus, JournalSeek, Journal Citation Reports/Science Edition, Directory of Open Access Journals
(DOAJ), African Journal Online, Bioline International, Open-J-Gate and Pharmacy Abstracts

\section{INTRODUCTION}

The prevalence of dementia has dramatically increased in ageing populations, affecting $1 \%$ of $60-64$ year olds, and up to $40 \%$ of those aged over 85 years [1]. According to the statistics from the World Health Organization (WHO), the incidence of dementia is increasing year by year. It was estimated that there were 35.6 million people with dementia worldwide in 2012, which may double in 2030 and more than triple in 2050. The Chinese population is rapidly aging. The sixth national population census showed that there were 178 million people aged over 60 years in China, accounting for $13.26 \%$ of the total population [1]. Therefore, dementia is becoming a challenge for patients, caregivers, and healthcare providers. Moreover, it brings a heavy financial burden, because the annual care cost ranges from $\$ 17,000$ to $\$ 55,200$ per patient.

Three types of dementias have been identified. These are Alzheimer's disease, vascular dementia and mixed dementia. [2] As the second most common form of dementia in the elderly, vascular dementia (VD) constitutes a heavy

(C) 2018 The authors. This work is licensed under the Creative Commons Attribution 4.0 International License 
burden to the health care systems in ageing societies [1]. VD is a degenerative disease caused by different vascular lesions, which restricts blood supply to individual brain regions [3]. It is usually associated with cognitive dysfunctions, as well as the impairments of memory and executive function [4]. Persistent reduction in cerebral blood flow induces hypoxia/ischemia of the brain tissue, and oxygen and nutrient deprivation contribute to cell death [5]. Alzheimer's dementia (AD) and VD appear to have similar clinical symptoms, such as attention deficits and action deficits. [6]. Moreover, specific cognitive domains are affected. However, it has been reported that there were significant variations among these subtypes. $A D$ is associated with better preserved initiative and performance than VD, according to the Interview for Deteriorations in Daily Living Activities in Dementia [2]. There are numerous studies focused on AD treatment, while VD has so far received very little attention.

Traditional vascular risk factors for VD are diabetes, hypercholesterolemia, hypertension and smoking. The association between cholesterol and small vessel disease (SVD), stroke, cognitive impairment and subsequent dementia is complicated and not yet fully elucidated. Moreover, the effect of lipids and lipid-lowering agents on the prevention or treatment of dementia is not fully understood. Studies on the effect of lipid-lowering therapy on the prevention (two trials) and treatment (four trials) of dementia found no evidence to support the use of lipid-lowering therapy [7].

Indeed, it was found that the lipid-lowering therapy was suitable for treating patients with vascular risk factors. It meet the criteria for lipidlowering therapy aimed at primary and secondary prevention of cardiovascular and cerebrovascular events, and it was recommended for managing patients in a holistic manner based on the vascular risk profiles [7]. Various drugs, including donepezil, galantamine, and memantine, approved for the treatment of $A D$, have shown modest cognitive benefits on VD patients. However, their functions and benefits were inconsistent. Donepezil showed some cognitive benefits in patients with VD only, while galantamine exhibited some advantages in mixed dementia (AD and VD).

The benefits of other drugs such as rivastigmine, memantine, nimodipine, and piracetam, were uncertain. Some other supplements and herbal therapies such as citicoline, actovegin, huperzine $A$ and vinpocetine have also been used on VD patients, but their beneficial effects were not well established. Non-drug therapies and lifestyle modifications such as diet, exercise and vascular risk factor control are important in the management of VD and should not be overlooked [8]. Acupuncture therapy stimulating the lesion area at the scalp effects. Although this treatment modality has been performed over 1000 years, it has advanced in recent years [9]. Moreover, in 1991 the WHO published the Standard International Acupuncture Nomenclature [10]. The effectiveness of scalp acupuncture for VD has been confirmed by a great deal of clinical trials [11-13].

Although the paucity of randomized control evidence is a challenge to clinical decision making, it provides multiple opportunities for ongoing and future researches. The treatment for dementia using traditional Chinese medicine (TCM) has a long history, and has proved effective and safe. Yizhi decoction has produced good effects in the treatment of VD. It contains Acorus calamus, Astragalus membranaceus and Polygala tenuifolia which had higher frequency of application in the treatment of VD. In order to evaluate the effectiveness of treatment of VD with Yizhi decoction, meta-analyses were carried out on related studies from 1995 to 2016.

\section{Data sources}

"Vascular dementia" and "Yizhi decoction" were chosen as the key terms used to retrieve information from several databases, including Chinese biomedical literature database, China master theses full-text database, China Journal Full-text Database, VIP database, and Chinese journals of TCM database. . A total of 262 publications were retrieved. After the exclusion of repeated literature and animal experiments, 57 papers were obtained for further screening.

\section{Establishment of inclusion and exclusion criteria}

The inclusion criteria of clinical randomized controlled trials for Yizhi decoction in the treatment of VD were based on the International Handbook of evidence-based medicine (Cochrane) collaboration network. Alzheimer's disease patients, severe dementia patients, pseudo depressive dementia or severe depression cases, and patients with senile psychosis, schizophrenia, epilepsy and Parkinson disease were excluded. In addition, patients with heart, lung, liver and kidney diseases; hypertension, diabetes and other diseases were excluded. . The diagnostic criteria of chemical medicine consisted of two parts, one was the Chinese expert consensus on the 
prevention and treatment of cognitive dysfunction, and the other one was based on the Diagnostic and Statistical Manual Spirit (4th edition), and the standard of the United States and the National Institute for Neurological Disorders and Stroke/Association Internationale pour la Recherche.

The diagnostic criteria of traditional Chinese medicine also contained two parts. One part, was the standard of diagnosis and the effective evaluation for VD reported by Tian Jinzhou [14]. The second one was the Chinese medicine treatment of dementia clinical research guideline for diagnosis mentioned in the second series of Guidelines of New Drug Clinical Medicine [15]. Randomized controlled trial (RCT) was used in the reported trials. A parallel control group was given placebo, no treatment or any other Western medicine. The balance between the groups was good and comparable. The treatment group was provided with Chinese medicine decoction. Finally, 18 articles did not accord with the diagnostic standard, 3 papers were not based on Yizhi Chinese herbal medicine, 7 were without RCT test, while 7 articles had Jadad score below 2 points. After further screening, 14 articles were found suitable for use in the study.

\section{Diagnostic criteria of effectiveness}

Total effectiveness, simple mental state rapid test table (MMSE), difference before and after treatment, and ability of daily living ability correction scale (ADL), before and after the treatment, were used for meta-analysis and effects assessment.

Table 1: Jadad score of the literature

\section{METHODS}

\section{Evaluation standard}

Jadad evaluation was used to determine literature quality, including random methods, random allocation concealment and blinding, withdrawal, exiting and other aspects. It was appropriate at 2 points, not clear at 1 point, and not appropriate at 0 point. High-quality literature was scored 3 points or higher, while low-quality literature was scored less than 3 points. Literatures with whose Jadad score less than 2 points were excluded.

\section{Quality assessment}

Rev man 5.3 provided by the Cochrne collaboration network was used for metaanalysis. Heterogeneity test between experimental results was processed first, and the test level was $p=0.05$. Random effects model and the fixed effects model were used when the test results appeared heterogeneous. FunnelPlot analysis was used for analyzing in the potential publication bias. Weighted by mean difference (WMD) was used as the index of measurement for continuous variables, while odds ratio (OR) was used for classified variables. The confidence interval $(\mathrm{Cl})$ used was $95 \%$.

\section{RESULTS}

\section{Literature screening}

A total of 262 publications were retrieved from 5 Chinese databases, and 57 papers were selected after repeated literature and animal experiments were excluded.

\begin{tabular}{|c|c|c|c|c|c|c|c|}
\hline No. & Literature & $\begin{array}{l}\text { Generation of } \\
\text { random } \\
\text { sequences }\end{array}$ & $\begin{array}{c}\text { Randomization } \\
\text { concealment }\end{array}$ & $\begin{array}{l}\text { Blind } \\
\text { method }\end{array}$ & $\begin{array}{l}\text { Withdrawal } \\
\text { and } \\
\text { withdrawal }\end{array}$ & $\begin{array}{c}\text { Baseline } \\
\text { equalization }\end{array}$ & $\begin{array}{l}\text { Jadadd } \\
\text { score }\end{array}$ \\
\hline 1 & Liu and Li, 2014 [16] & visiting order & - & $\overline{-}$ & - & comparable & 2 \\
\hline 2 & Li et al 2013 [17] & admission time & - & - & - & comparable & 2 \\
\hline 3 & Li et al 2013 [18] & admission time & - & - & - & comparable & 2 \\
\hline 4 & Li and Wang 2016 [19] & Stochastic & - & - & - & comparable & 2 \\
\hline 5 & Gao and Liu 2015 [20] & visiting order & pharmacy & - & - & comparable & 4 \\
\hline 6 & Sun et al 2015 [21] & Stochastic & pharmacy & - & - & comparable & 3 \\
\hline 7 & Zhan et al 2016 [22] & Stochastic & pharmacy & - & - & comparable & 4 \\
\hline 8 & Li et al 2013 [23] & admission time & - & - & - & comparable & 2 \\
\hline 9 & Huang 2007 [24] & Stochastic & - & - & - & comparable & 2 \\
\hline 10 & Qin and Wei 2007 [25] & visiting order & - & - & - & comparable & 2 \\
\hline 11 & Wang 2011 [26] & Stochastic & pharmacy & - & - & comparable & 3 \\
\hline 12 & Zhao et al 2015 [27] & Stochastic & - & - & + & comparable & 5 \\
\hline 13 & Miao 2006 [28] & Stochastic & pharmacy & - & - & comparable & 3 \\
\hline 14 & Qu 2013 [29] & Stochastic & - & - & - & comparable & 2 \\
\hline
\end{tabular}


Table 2: Basic statistics of literature outcome indicators

$$
\begin{aligned}
& \text { No. Literature }
\end{aligned}
$$

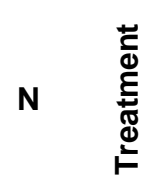

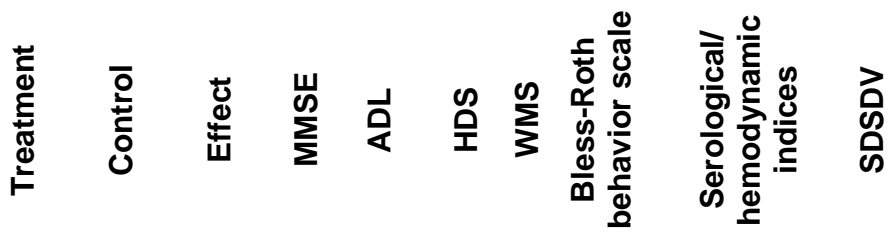

\begin{tabular}{|c|c|c|c|c|c|c|c|c|c|c|c|}
\hline 1 & Liu and Li, 2014 [16] & 78 & 39 & 39 & + & + & + & & & & + \\
\hline 2 & Li et al 2013 [17] & 48 & 24 & 24 & + & + & + & & + & + & \\
\hline 3 & Li et al 2013 [18] & 46 & 23 & 23 & + & + & + & & & + & \\
\hline 4 & Li and Wang 2016 [19] & 40 & 20 & 20 & + & + & + & & & & \\
\hline 5 & Gao and Liu 2015 [20] & 60 & 30 & 30 & & + & + & & & & + \\
\hline 6 & Sun et al 2015 [21] & 86 & 43 & 43 & + & + & + & + & + & + & \\
\hline 7 & Zhan et al 2016 [22] & 115 & 58 & 57 & + & + & + & + & & & \\
\hline 8 & Li et al 2013 [23] & 70 & 35 & 35 & + & + & + & & & + & \\
\hline 9 & Huang 2007 [24] & 76 & 38 & 38 & + & + & + & & & & \\
\hline 10 & Qin and Wei 2007 [25] & 61 & 33 & 28 & + & + & + & + & & & \\
\hline 11 & Wang 2011 [26] & 83 & 42 & 41 & + & & & & & & \\
\hline 12 & Zhao et al 2015 [27] & 102 & 52 & 50 & & + & + & & & + & + \\
\hline 13 & Miao 2006 [28] & 60 & 30 & 30 & & + & & & & & \\
\hline 14 & Qu 2013 [29] & 120 & 60 & 60 & + & & & & & + & \\
\hline
\end{tabular}

Through inclusion and exclusion criteria, 14 RCT were finally chosen, including 6 high quality RCT articles and 8 low-quality RCT articles. Total efficiency was recorded in 11 articles, curative effect of TCM syndrome was reported in 3 articles, MMSE score was reported in 12 articles, and ADL was mentioned in 11 articles. Moreover, Kyohko Hasegawa Dementia Scale was used in 3 articles, WMS score was recorded in 2 articles, while blood flow index was mentioned in 6 articles. There was a total of 1045 patients, including 527 cases treated with Yizhi decoction group, and 518 cases in the control group. These details are shown in Table 2.

\section{Determination of publication bias}

Funnel plots produced using Revman 5.3 analysis software were used to detect publication bias. Results showed absence of publication bias. Therefore, the conclusions were reliable.

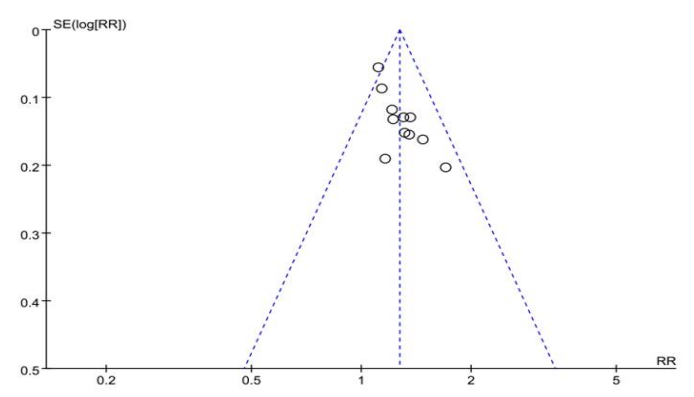

Figure 1: Funnel diagram of general therapeutic effect of Yizhi decoction on VD

\section{Results of meta-analysis of total effectiveness}

A total of 14 papers were screened, and 11 of them reported the total effectiveness of Yizhi decoction in the treatment of VD. Meta-analysis was carried out on 11 observational data, and the results are shown in Figure 2. The total effectiveness for the studies showed homogeneity (heterogeneity test $=10.88, p$ $=0.37$ ). Thus, the Risk Ratio (RR) of the combined effect was analyzed usingthe fixed effect model. The RR merger was 1.27, and the 95\% confidence interval was between 1.18 and 1.37, indicating that the $R R$ value was statistically significant, which suggest that Yizhi decoction was effective in the treatment of VD.

\section{Meta-analysis results for MMSE}

A total of 12 studies measured MMSE before and after treatment, and 11 descriptive methods were used to describe the differences among MMSE scores before and after treatment. These 11 articles were used to study the change of MMSE score after the treatment with weighted mean difference. The results (Figure 4) indicated that the 11 items had heterogeneity (heterogeneity test $=19.93, p=0.03$ ). Therefore, the combined effect of the WMD was analyzed using a fixed effects model. The WMD was 3.6, and the $95 \%$ $\mathrm{Cl}$ was between 3.14 and 4.07, which indicated that the WMD value was statistically significant. 


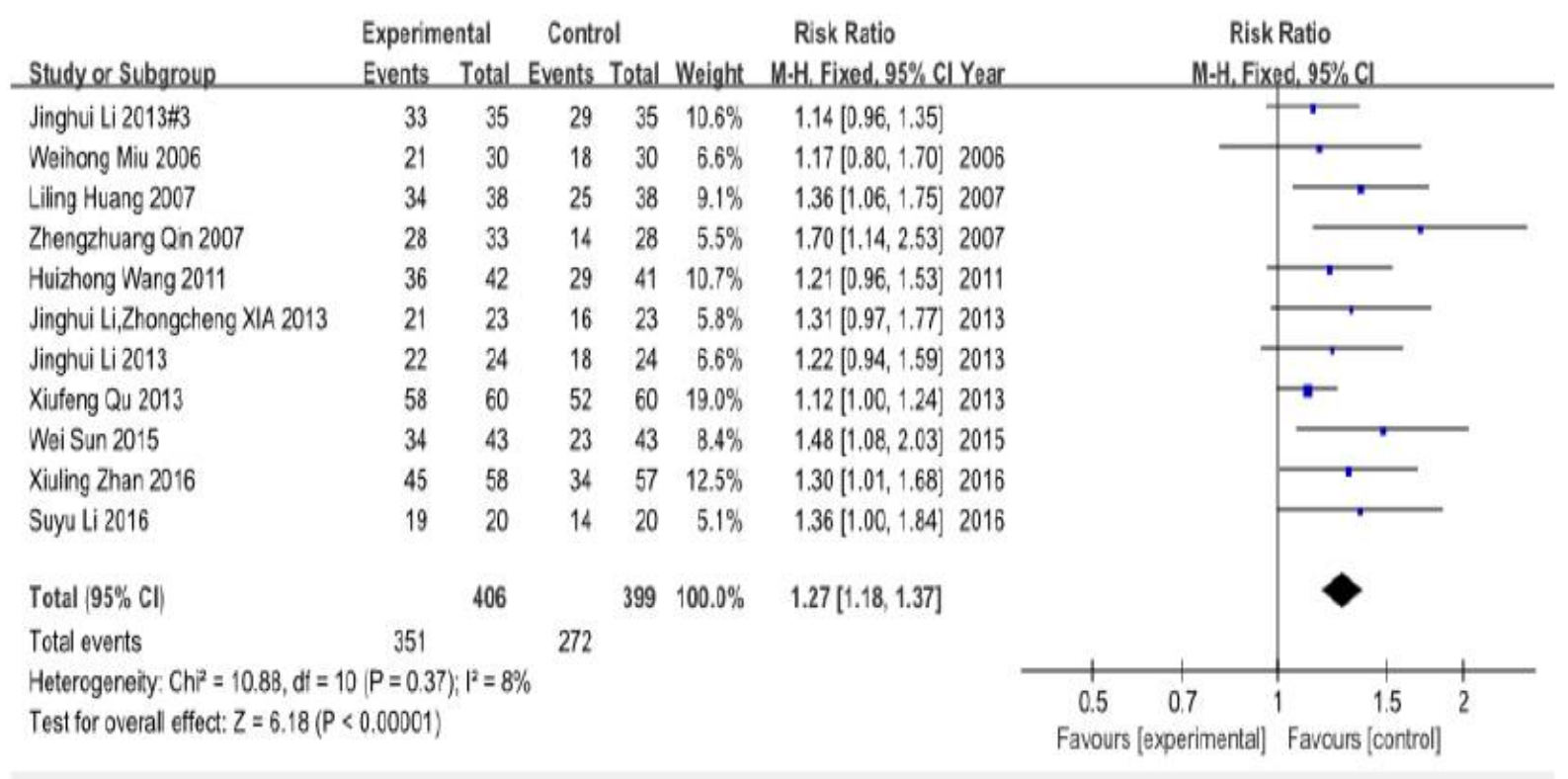

Figure 2: Forest diagram of the therapeutic effect of Yizhi decoction on VD

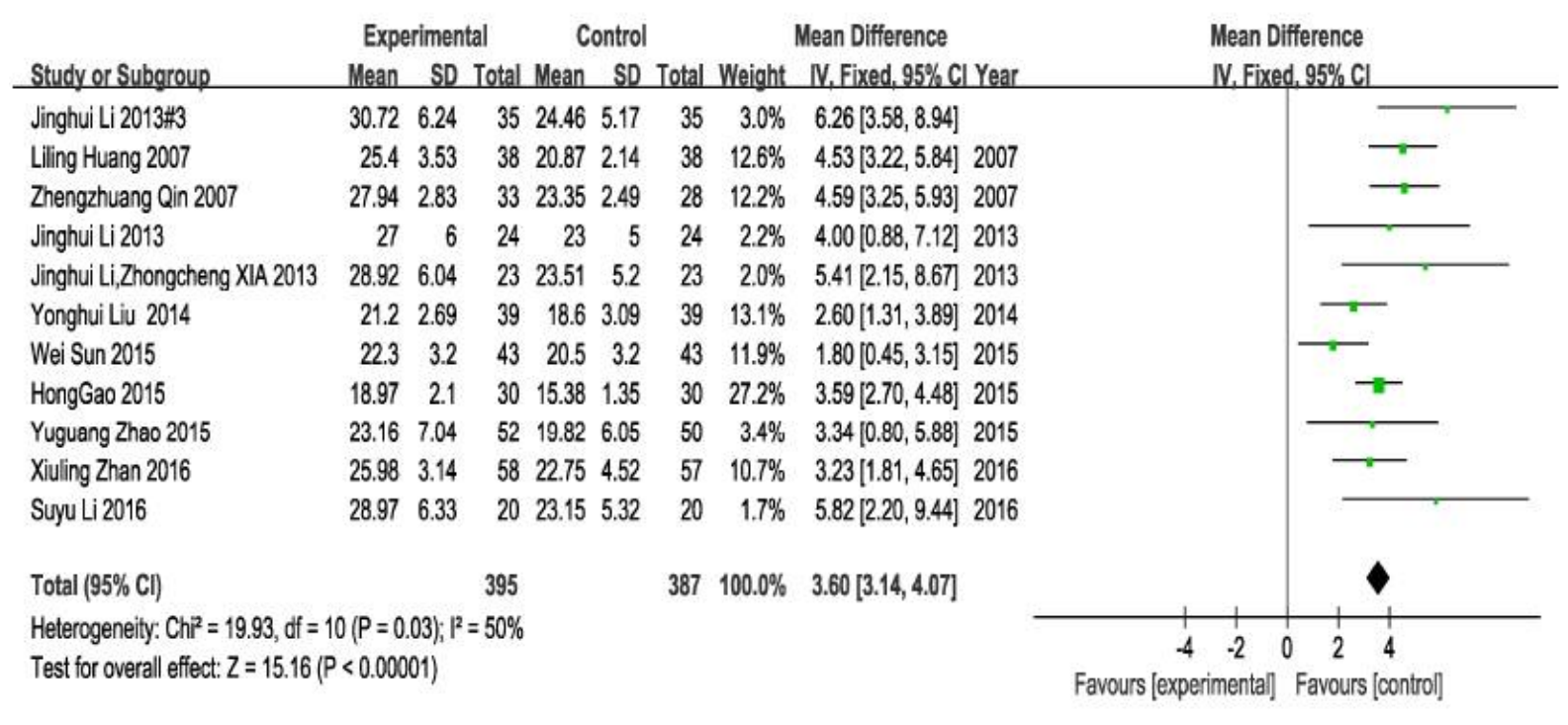

Figure 3: Forest chart of simple mental state quick check list

Thus, it was inferred that Yizhi decoction improved the MMSE score of patients with VD.

\section{Meta-analysis of ADL scores}

ADL scores before and after treatment were reported in 11 studies, and 10 studies which referred to the differences were analyzed. The 10 studies were with homogeneity (heterogeneity test $=46, p<0.00001)$. Therefore, the analysis of the combined effect of IR used a random effects model. The IR was 1.44 , its $95 \%$ confidence interval was between 5.72 and 2.84, which indicated that the IR value was statistically significant. Sensitivity analysis (Figure 3) indicated that the result was stable and reliable.

\section{Subgroup analysis}

\section{Based on time of administration}

Subgroup analysis included two groups: within 30-days group (including the 30 days, regarded as short-term medication group) and more than 30-days group (regarded as long-term medication group). The analysis was conducted based on the MMSE scores. There were 5 articles for short-term medication, and 9 articles for long-term medication. The MMSE scores were reported in 6 articles of long-term medication, and $\mathrm{I}^{2}$ (index of heterogeneity) was $97 \%>50 \%$. This indicated that the analyzed subjects were heterogeneous. Therefore, the random effects model was used to analyze. 


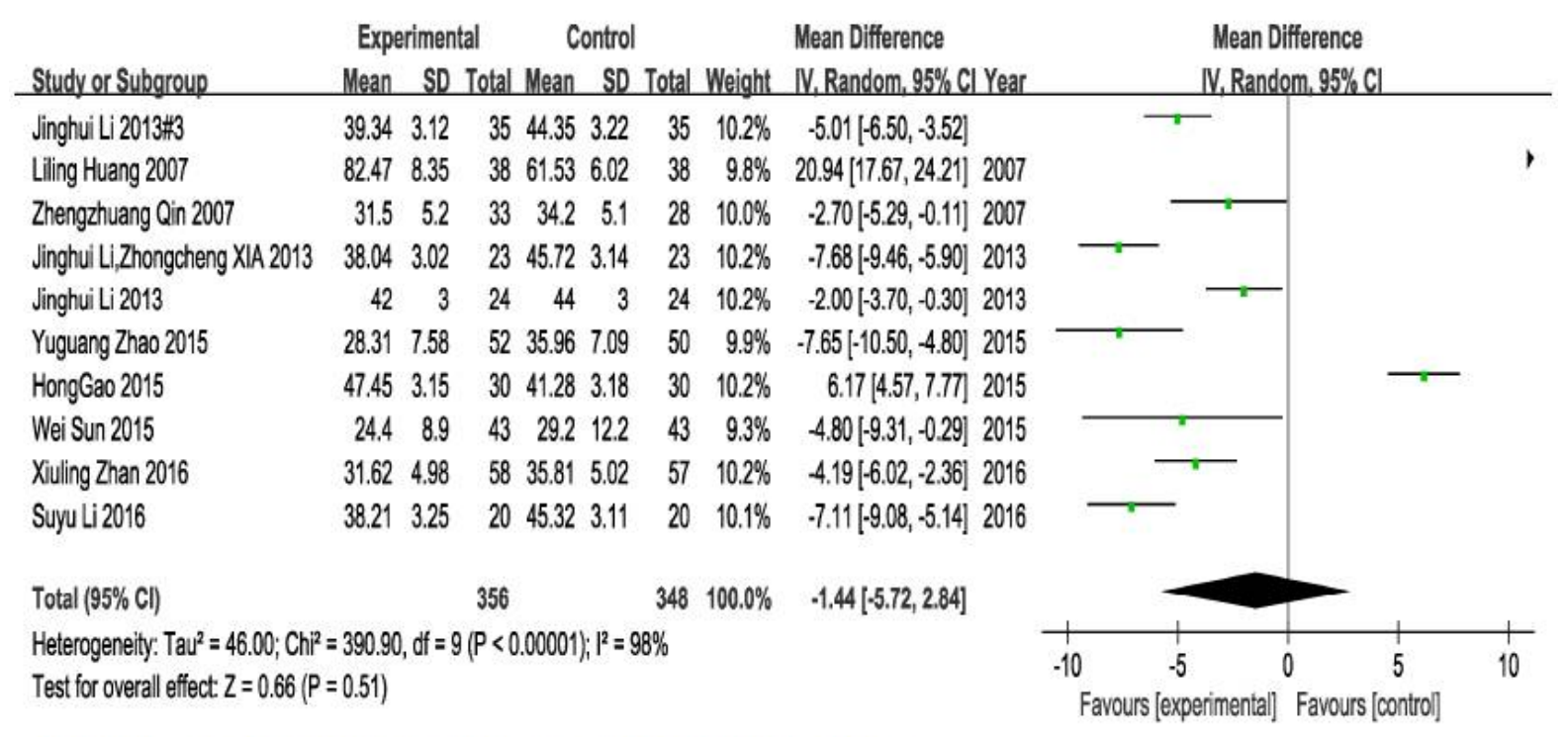

Figure 4: Forest map of correction scale score of daily life ability

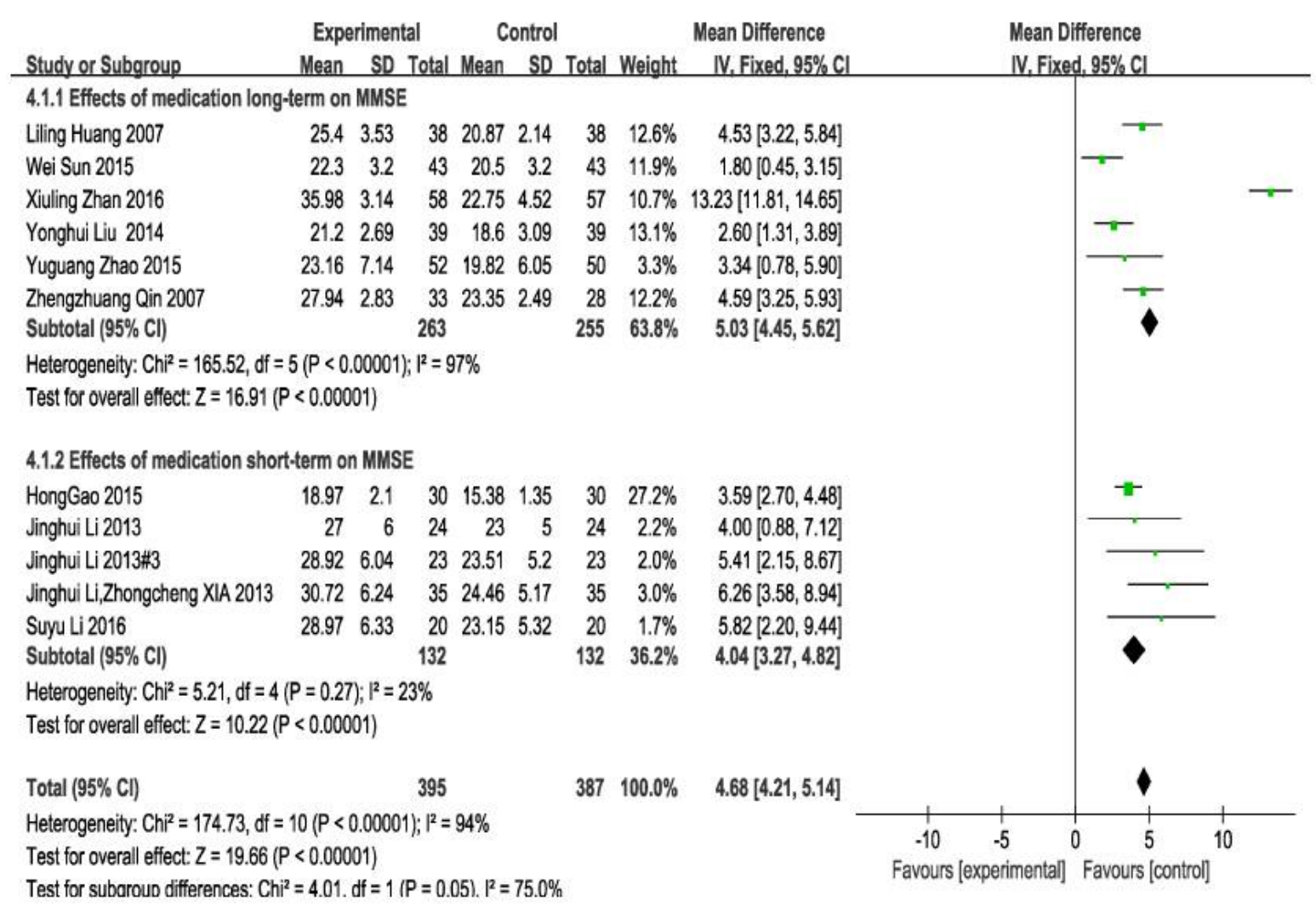

Figure 5: Forest map of subgroup analysis for MMSE score of time

Merged MD value amounted 5.03, the 95\% confidence interval was between 4.45 and 5.62 , and the overall effect test $Z$ was 16.91, $p<0.05$. There were 5 articles on short-term medication reported MMSE scores, and $\mathrm{I}^{2}$ was $23 \%<50 \%$, which meant that the 5 subjects were with homogeneity. Thus, the fixed effect model was used. The rhombus was at the right of the vertical; merged MD value was 4.04, and the 95 $\%$ confidence interval was between 3.27 and
4.82. The overall effect test $Z$ was 10.22, ( $p<$ 0.05 ) and the result of MMSE score is shown in Figure 5.

The subgroup analysis was used for two ADL key evaluation indices, and groups were divided by MMSE scores. There were 5 articles for longterm medication, and the $\mathrm{I}^{2}$ (index of heterogeneity) was $98 \%>50 \%$, which indicated the analyzed subjects were heterogeneous. Therefore, the random effects model was used

Trop J Pharm Res, December 2018; 17(12): 2502 


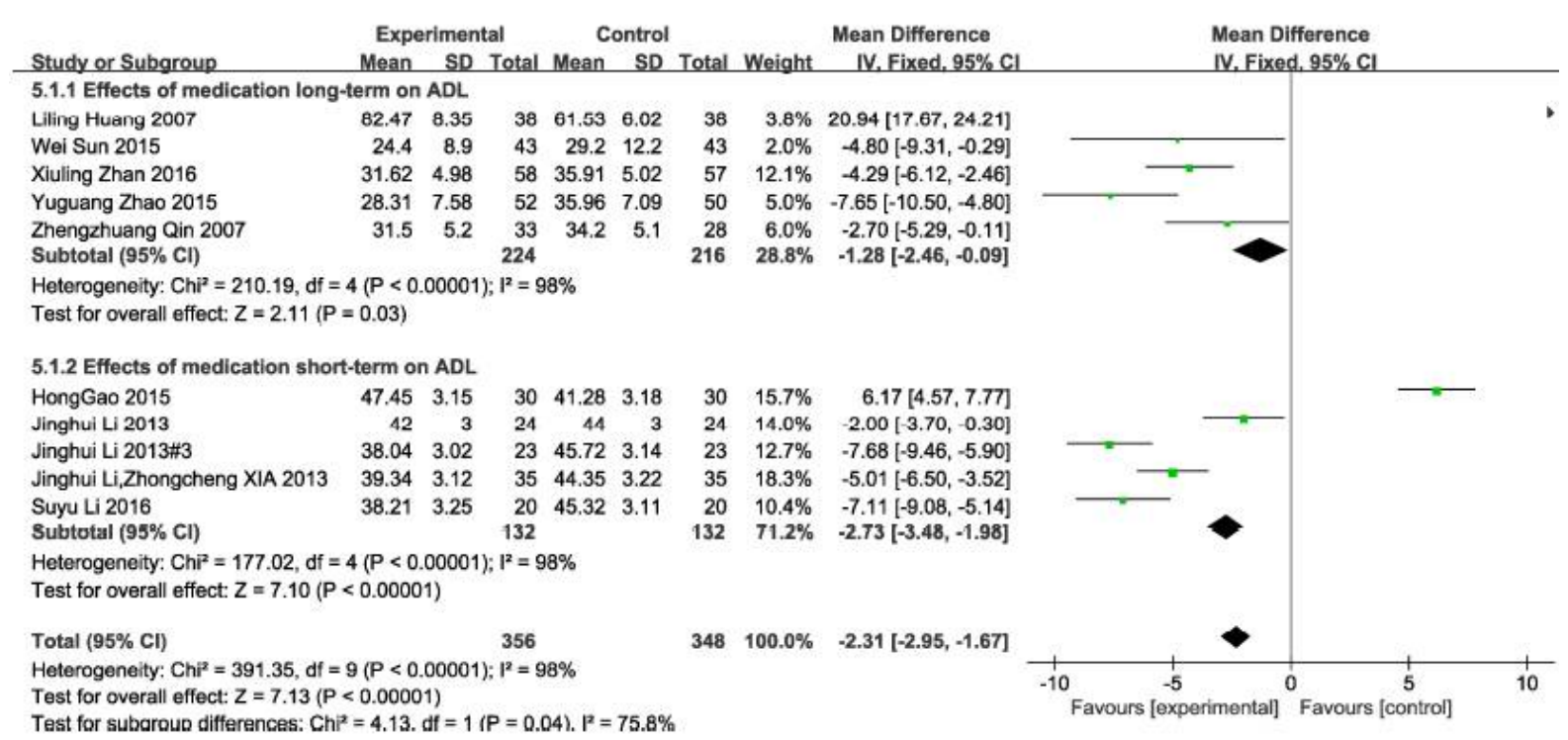

Figure 5: Forest map of subgroup analysis for ADL score of time

for analysis. Merged MD value was -1.28 , with $95 \%$ confidence interval between -2.46 and 0.09 , and the overall effect test $Z$ was 2.11 ( $p<$ 0.05). There were 5 articles on short-term medication that reported ADL scores. The $\mathrm{I}^{2}$ was $98 \%>50 \%$, which meant that the 5 subjects were heterogeneous. Thus, the random effects model was used. The rhombus was at the left of the vertical, and merged MD value was -2.73 ; the $95 \%$ confidence interval was between -3.48 and -1.98, and the overall effect test $Z$ was 7.1 ( $p<$ $0.05)$. The result of analysis of ADL scores is shown in Figure 5.

\section{Based on intervention method}

Groups were divided into those administered Yizhi alone and those administered Yizhi in combination. The analysis was conducted based on the MMSE scores. It could be seen that 7 articles on Yizhi administered alone reported MMSE scores, and $\mathrm{I}^{2}$ was $55 \%>50 \%$, which showed that the analyzed subjects were with heterogeneity. Thus, the random effects model was used for analysis. Merged MD value amounted to 3.44 , with $95 \%$ confidence interval between 2.83 and 4.05 , and the overall effect test $Z$ was $11.09(p<0.05)$. There were 4 articles on Yizhi administered in combination, and they reported MMSE scores. The $\mathrm{I}^{2}$ was $50 \%$, which implied that the subjects included in the study had homogeneity, and the fixed effect model was used for analysis. The rhombus was at the right of the vertical, merged MD value was 3.84, the $95 \%$ confidence interval was between 3.11 and 4.56, and the overall effect test $Z$ was 10.37 ( $p<$ $0.05)$. The results are shown in Figure 6.
The subgroup analysis was for two ADL key evaluation indices, and groups were divided based on MMSE scores. It could be seen that there were 7 articles on the Yizhi administered alone that reported ADL scores, and $I^{2}$ was $97 \%>50 \%$. It indicated that the analysis object had heterogeneity, so the random effects models was for analysis. Merged MD value was -2.98, with $95 \%$ confidence interval between -3.67 and -2.30, and the overall effect test $Z$ was 8.5 ( $p<$ 0.05). There were 3 articles on Yizhi administered in combination, with ADL scores, and the $\mathrm{I}^{2}$ was $99 \%$, meaning that the subjects included in the studies had heterogeneity. Thus, the fixed effect model was used. Merged MD value was 1.75; the $95 \%$ confidence interval was between 0.09 and 3.14 , and the overall effect test $Z$ was $2.06(p<0.05)$. These results are shown in Figure 7.

\section{DISCUSSION}

This study has shown that Yizhi decoction improved the quality of daily life and mental state of VD patients, on the basis of the results from clinical total effectiveness, and the ADL and MMSE meta-analysis. There are several limitations in this study. Firstly, a few foreign data were we disregarded. This might be one of the reasons for publication bias in the metaanalyses. Secondly, due to limited or missing data on subsets in the current trials, there are still several details missing. It was not possible to assess the long-term curative effect and the influence on the life quality and the mortality for VD patients on Yizhi decoction therapy. Because all the studies did not report the quality of living of the patients, follow-up and mortality of patients after treatment are not known. The evaluation 


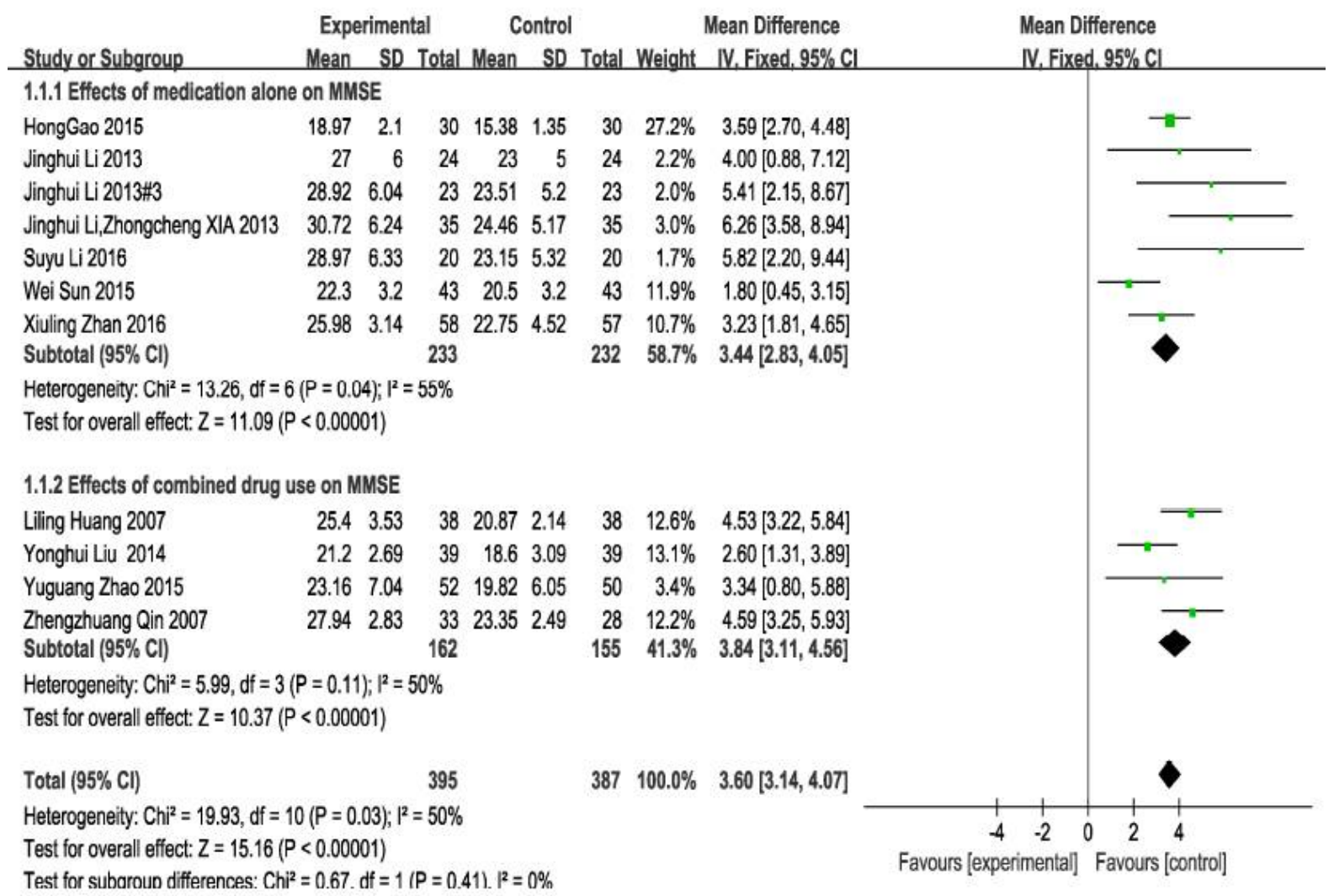

Figure 6: Forest map of subgroup analysis for MMSE score of intervention method

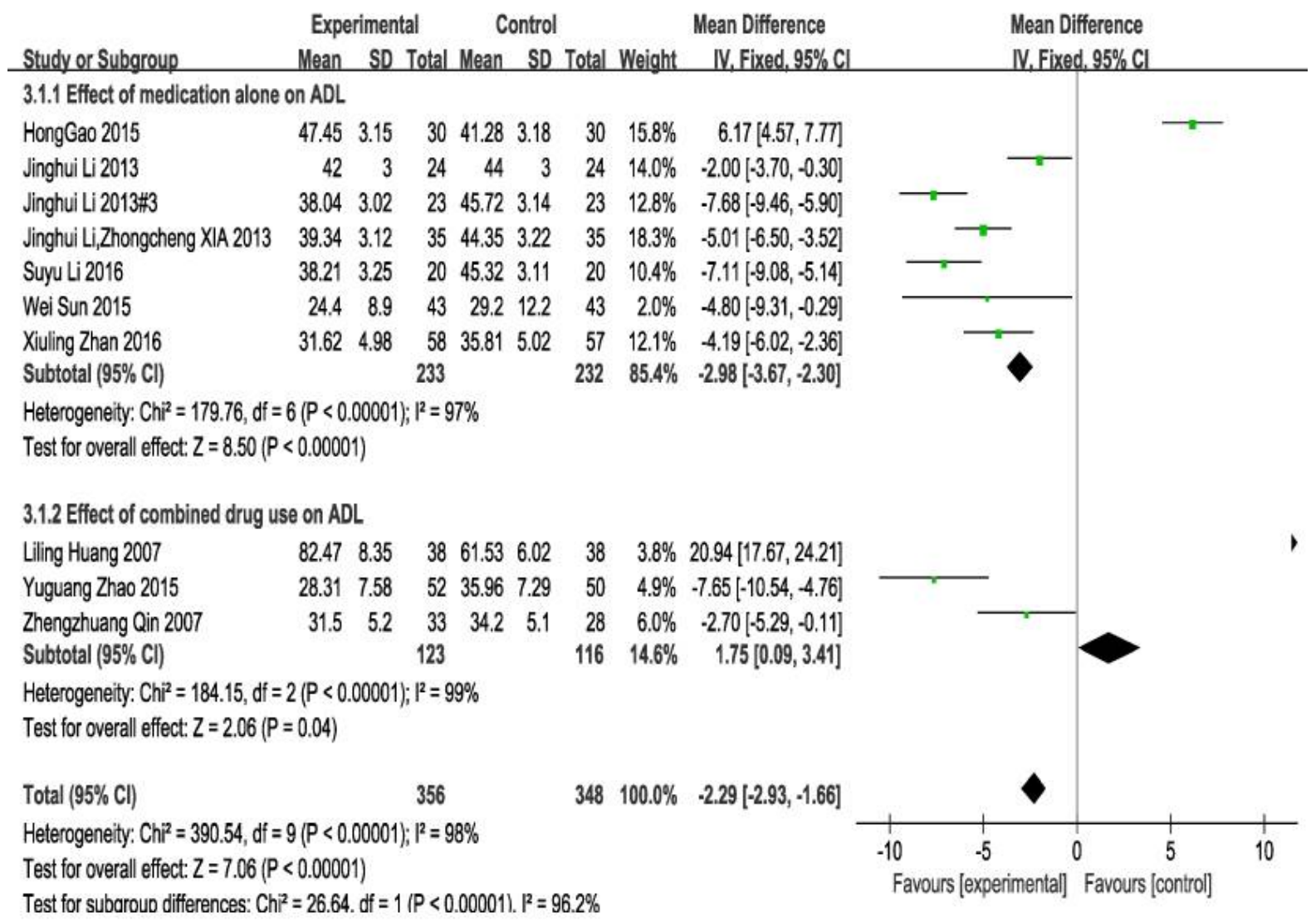

Figure 7: Forest map of subgroup analysis for ADL score of intervention method 
system was low quality, the literature description of randomization was not clear enough, and most randomized allocation schemes were not hidden. Moreover, most of the literature were not blinded, and there were midway withdrawals. These factors limited the credibility and effectiveness in the treatment group. The literature had different treatment methods for the control group, and the sample included in articles was small. Therefore, larger sample studies with more rigorous scientific evaluations are needed for further confirmation.

\section{CONCLUSION}

Yizhi decoction improves the quality of daily life and mental state in VD patients. However, more rigorous and scientific case studies are still needed for further confirmation to strengthen and support these findings.

\section{DECLARATIONS}

\section{Acknowledgement}

The authors thank the reviewers for their critical comments on the manuscript.

\section{Conflict of Interest}

No conflict of interest associated with this work.

\section{Contribution of Authors}

The authors declare that this work was done by the authors named in this article and all liabilities pertaining to claims relating to the content of this article will be borne by them.

\section{REFERENCES}

1. Zhang LG, Wang LJ, Shen QQ, Wang HF, Zhang Y, Shi GG, Zhang SC, Zhang MY. Paeoniflorin improves regional cerebral blood flow and suppresses inflammatory factors in the hippocampus of rats with vascular dementia. Chin J Integr Med 2017; 23: 696702.

2. Giebel CM, Burns A, Challis D. Taking a positive spin: preserved initiative and performance of everyday activities across mild Alzheimer's, vascular and mixed dementia. Int J Geriatr Psychiatry 2017; 32: 959-967.

3. Gupta S, Sharma B. Pharmacological modulation of I 1 imidazoline and a 2-adrenoceptors in subacute brain ischemia induced vascular dementia. Eur J Pharmacol 2014; 723: 80-90

4. Simard M, van Reekum R, Cohen T. A review of the cognitive and behavioral symptoms in dementia with Lewy bodies. J Neuropsychiatry Clin Neurosci 2000; 12 : 425.
5. Reischl S, Li L, Walkinshaw G, Flippin LA, Marti HH, Kunze R. Inhibition of HIF prolyl-4-hydroxylases by FG4497 reduces brain tissue injury and edema formation during ischemic stroke. Plos One 2014; 9: e84767.

6. Arnaoutoglou NA, Arnaotoglou M, Nemtsas P, Costa V. Color perception differentiates Alzheimer's Disease (AD) from Vascular Dementia (VaD) patients. Int Psychogeriatr 2017; 29: 1355-1361.

7. Appleton JP, Scutt $P$, Sprigg $N$, Bath PM. Hypercholesterolaemia and vascular dementia. Clin Sci2017; 131: 1561-1578.

8. Farooq MU, Min JY, Goshgarian C, Gorelick PB. Pharmacotherapy for vascular gognitive impairment. CNS Drugs 2017; 31: 759-776.

9. Liu Z, Guan L, Wang Y, Xie CL, Lin XM, Zheng GQ. History and mechanism for treatment of intracerebral hemorrhage with scalp acupuncture. Evid Based Complement Alternat Med 2012; 2012: 895032.

10. WHO Scientific Group on International Acupuncture Nomenclature. A proposed standard international acupuncture nomenclature: report of a WHO scientific group. Geneva: World Health Organization; 1991.

11. Wang Y, Shen J, Wang XM, Fu DL, Chen CY, Lu LY, et al. Scalp acupuncture for acute ischemic stroke: a metaanalysis of randomized controlled trials. Evid Based Complement Alternat Med 2012; 2012: 480950.

12. Lee GE, Yang HD, Heo EJ, Jeon WK, Lyu YS, Kang HW. The current state of clinical studies on scalp acupuncture-treatment for dementia-by search for China literature published from 2001 to 2011 in CAJ (China Academic Journals). J Orient Neuropsychiatry. 2012; 23: 13-32.

13. Peng W, Zhao H, Liu Z, Liu B. Systematic assessment of electroacupuncture treatment for vascular dementia. Chin Acupunct Moxibustion. 2004; 24: 297-301.

14. Tian JZ, Han MX, Tu JW. The diagnosis, dialectical and efficacy criteria for vascular dementia. Journal of Beijing University of Traditional Chinese Medicine, 2003, 23: 16-24.

15. Ministry of Health of the PRC. Guidelines for treatment of dementia with new Chinese Medicine. Beijing: People's Medical Publishing House.1995:206-210.

16. Liu YH, Qi HM. Efficacy observation on treating vascular dementia with the Bushen Anshen Yizhi decoction. Clinical Journal of Chinese Medicine 2014; 6: 17-19.

17. Li JH, Gui CL, Xia ZC. Clinical investigation of Bushen Yizhi decoction on vascular dementia. Shanxi Medicine J 2013; 2: 168-170.

18. Li JH, Xia ZC, Gui CL. Therapeutic effects of Bushen Yizhi decoction combined with GM1 on vascular dementia. Liaoning $J$ Traditional Chinese Medicine 2013; 40: 481-483.

19. Li SY, Wang JL. Clinical observation on treating vascular dementia with the Bushen Yizhi decoction plus ganglioside. Clinical Journal of Chinese Medicine 2016;8:95-96.

Trop J Pharm Res, December 2018; 17(12): 2505 
20. Gao H, Liu WH. Clinical investigation of Shenqi Yizhi decoction on 60 cases of vascular dementia. Guang Ming Zhong Yi 2015; 5: 992-994.

21. Sun W, Zhang Q, Yang JB, Yang JF, Zhang ZZ. Clinical effects of Gunao Yizhi decoction combined with donepezil in treating the senile kidney deficiency and phlegm turbid type vascular dementia. Chinese $J$ Gerontol 2015; 1: 84-86.

22. Zhan XL, Fan GF, Han BZ, Wang ZQ, Liu W, Jiao CC. Clinical investigation of Gunao Yizhi decoction combined with donepezil in treating the senile kidney deficiency and phlegm turbid type vascular dementia. Modern $J$ Integrat Tradit Chinese Western Medicine 2016; 25: 3012-3014.

23. Li JH, Cai LL, Li DM, Xia ZC. Clinical efficacy observation on treating senile vascular dementia with Huatan Quyu Yizhi decoction. J Sichuan Tradit Chinese Medicine 2013; 31: 73-75.

24. Huang LL. Efficacy observation on treating vascular dementia with Jiannao Yizhi decoction. Clin J Trad Chinese Medicine 2007; 19: 366-367.
25. Qin ZZ, Wei JS. Efficacy observation of Senile Yizhi decoction on 33 cases of senile vascular dementia. Zhejiang Journal of Traditional Chinese Medicine 2007; 42: 208-209.

26. Wang HZ. Efficacy observation on treating vascular dementia with Lingnao Yizhi decoction. JCM 2011; 9: 41-42.

27. Zhao YG, Li FS, Li XP, Qiao QZ, Guo QH. Observation of the clinical efficacy of Shuanghuang Yizhi decoction on vascular dementia. World J Integrat Tradit Western Medicine 2015; 10: 508-510.

28. Miao WH. Clinical observation on treating vascular dementia with Yizhi decoction. Liaoning J Tradit Chinese Medicine 2016; 33: 1067.

29. Qu XF, Huang YQ. Observation of the combination of Chinese Traditional and Western Medicine on 60 cases of vascular dementia. J Pract Tradit Chinese Medicine 2013; 2: 105-106. 